\title{
Standardised methods for amino acid analysis of food
}

\author{
Don E. Otter* \\ Food Nutrition Genomics Team, Agri-Foods \& Health Section, Grasslands Research Centre, AgResearch Limited, \\ Palmerston North, New Zealand
}

(Submitted 26 July 2011 - Final revision received 21 November 2011 - Accepted 13 December 2011)

\begin{abstract}
Amino acids (AA) are essential nutritional components of a balanced diet and occur in foods in either the free AA form or as the building blocks of proteins. The analysis of AAs in foods is composed of a number of unit operations; the release of the AAs from the food matrix, the separation of the individual AAs and their quantification using calibration standards. Each of these steps has their own idiosyncrasies, e.g. different hydrolysis conditions are required for the optimal release of different AAs and there are a diverse number and type of food matrices, such that most laboratories adapt methods to best suit their applications. There is currently no official standardised method for AA analysis, although the Association of Analytical Communities (AOAC) has validated methods for a number of individual AA components. The established analytical techniques of HPLC (ion exchange or reversed phase) and GC-MS have recently been supplemented by a number of new methods. These include capillary electrophoresis MS and Ultra HPLC-MS, and LC with other detectors. This review will address the intricacies and concerns of the protein hydrolysis step, discuss what specifications or prerequisites need to be placed on the existing and new methods and laboratories using these methods, comment on whether one method can successfully satisfy the exacting requirements of the various unit operations, and finally pose the question 'Is there any merit in 'developing' a validated (e.g. AOAC) official method of analysis for AAs in food?'
\end{abstract}

\section{Key words: Amino acid: Analysis: Quantification: Food}

Growth in the world population has led to an unprecedented demand for quality food protein. Protein quality can be defined as the nutritional adequacy of food proteins to support both growth and health, and is now usually assessed through analysis of the amino acid (AA) composition of the protein. Thus it is essential that AA compositions be accurately determined. The analysis of the AA content of protein can be divided into a number of unit operations:

(1) Hydrolysis of individual AAs from the protein backbone

(2) Separation of individual AAs using a chromatographic procedure

(3) Detection and quantification of the separated AAs.

For any discussion on the analysis of AAs the key defining factors are accuracy, suitability and accountability. Any analysis should include performance data for each operation and the sum of the operations, and should also include appropriate quality controls, both internal and external, to ensure consistency and accuracy of results.

New methods for analysing AAs are continually being developed and are driven by speed of analysis (throughput), sensitivity, robustness and reproducibility. These advances have been aided by progress in instrumentation, e.g. MS, and derivatisation chemistry. There is also considerable cross over with the advancements in the fields of proteomics and metabolomics, such that the methods reviewed will not be restricted to the analysis of AAs in food, but to all new developments in AA analysis.

As the analysis of AAs in food products has been well served by some excellent reviews during the last decade ${ }^{(1,2)}$, this review will focus on highlighting recent developments in the analysis of AAs, with specific emphasis on foods, it will address the current state of play with regard to regulatory issues, and finally, it will outline the pros and cons of instigating standardised AA analysis methods for the food industry.

\section{Protein hydrolysis}

The release of the individual AAs from the protein backbone (protein hydrolysis) has been the 'poor cousin' with respect to optimising the individual unit operations described above. For the majority of AA analysis methods the basic operation

Abbreviations: AA, amino acid; AOAC, Association of Analytical Communities (officially known as Association of Official Agricultural Chemists although since 1991 informally known as Association of Analytical Communities); LC, liquid chromatography; LOQ, limit of quantification. 
of hydrolysing the peptide bond between adjoining AAs using $6 \mathrm{M} \mathrm{HCl}$ under vacuum and heating $\left(100-165^{\circ} \mathrm{C}\right)$ for up to $72 \mathrm{~h}$ has remained largely unchanged since $1954^{(3)}$. The research described below has gone some way to redress this imbalance and helps to address the perceived weaknesses of the hydrolysis step such as the long hydrolysis times, low throughput and the inherent instability of some AAs. The advent of new proteomic methods may also transfer over to AA analysis and offer opportunities for novel technology developments. Other possible protein hydrolysis methods that have not received much attention include the use of enzymatic cleavage of peptide bonds using a range of broad spectrum proteases and will not be discussed in this review.

It has long been recognised that the standard hydrolysis conditions are not suitable for the extraction of all AAs as some AA are unstable under these conditions, e.g. sulphur AAs (cysteine and methionine) and tryptophan, and this has resulted in varying hydrolysis conditions for these exceptions. Researchers at Massey University have been at the forefront of recent work to fully characterise the hydrolysis conditions required for optimal recovery of the individual AAs in different food products and feedstuffs ${ }^{(4,7)}$. Their work succinctly illustrates how some AAs can be either under or overestimated during standard acid hydrolysis conditions, and also offers insights into methods, both practical and by using modelling, to circumvent these difficulties.

An automatic protein hydrolysis system has been developed using strong cation-exchange resins that worked as solid acid catalysts for protein hydrolysis when heated in the presence of water $^{(8)}$. More work is still required to optimise the method as the most efficient resin of the inorganic solid acids and cationexchange resins tested yielded amounts of AAs that were only $70-75 \%$ of those recovered after conventional hydrolysis with $\mathrm{HCl}$, although the AA compositions closely matched the theoretical values. The hydrolysis temperature and time $\left(110^{\circ} \mathrm{C}\right.$ for $\left.20 \mathrm{~h}\right)$ were similar to conventional protein hydrolysis, but protein contamination was minimised by connecting the columns directly into a HPLC system, after heating them.

In 'real' food systems, rates of hydrolysis can also be affected by other constituents. The effect of different fat content $(30,45$ and $60 \%(\mathrm{w} / \mathrm{w})$ fat in $\mathrm{DM})$ in bovine casein and in model-processed cheeses on the hydrolysis curves of fifteen AAs were obtained by means of nonlinear regression using thirteen hydrolysis intervals (temperature, $110^{\circ} \mathrm{C}$; time, $0-144 \mathrm{~h})^{(9)}$. Correction factors that increased with the fat content were calculated to increase the accuracy of the AA determinations with the highest correction factors required for threonine, serine and tyrosine.

The principal concerns with these methods are the long hydrolysis times, and that the conditions should ideally be optimised for every AA and food type. Some of these parameters were addressed for milk in a study that compared six combinations of hydrolysis agent and temperature-time conditions with an Association of Analytical Communities (AOAC) reference method ${ }^{(10)}$. A rapid hydrolysis method using $6 \mathrm{~N} \mathrm{HCl}$ at $160^{\circ} \mathrm{C}$ for $60 \mathrm{~min}$ resulted in no statistically differences when compared with the reference method and was characterised by high precision, low repeatability uncertainty, and high accuracy for all AAs evaluated; the recovery mean value of the single AA was $98.4 \%$.

Microwave-assisted acid hydrolysis has been explored as one method to speed up the hydrolysis. Hydrolysis times, temperatures and sample weights were optimised for the microwave acid hydrolysis $(6 \mathrm{~N} \mathrm{HCl})$ of whole grain wheat $^{(11)}$. The hydrolysis parameters of $150^{\circ} \mathrm{C}, 3 \mathrm{~h}$ and $200 \mathrm{mg}$ sample weight represented an optimal balance between liberating AA residues from the wheat matrix and limiting their subsequent degradation or transformation, with a total AA recovery corresponding to at least $85 \cdot 1 \%$ of the total protein content.

In a more specific application, meat and meat-based products were subject to a $20 \mathrm{~min}$ microwave hydrolysis time to liberate 4-hydroxyproline to the same precision and accuracy as traditional hydrolysis methods. There was a reduction in hydrolysis time from $24 \mathrm{~h}$ and, although the other AAs were not quantified, visual examination of the chromatograms suggested similar levels of precision. Amino acid separation and detection was by high performance anion exchange chromatography and pulsed amperometric detection which allowed detection of 4-hydroxyproline without pre- or postcolumn derivatisation ${ }^{(12)}$.

Faster hydrolysis times were achieved using strongly microwave absorbing silicon carbide-based microtiter platforms (four platforms of twenty standard liquid chromatography (LC)/GC vials) which allowed for the high-throughput simultaneous hydrolysis of eighty samples with a 5 min irradiation at $160^{\circ} \mathrm{C}$. Only small amounts of sample $(25 \mu \mathrm{g})$ and solvent $(100 \mu \mathrm{l}$ of $6 \mathrm{~N} \mathrm{HCl})$ were required and transfer errors were minimised as one vial was used throughout the entire analysis $^{(13)}$.

In summary, significant advances have been made in fully characterising the protein hydrolysis step but there has been no sea change moment or method that is likely to displace the established $6 \mathrm{~N} \mathrm{HCl}, 110^{\circ} \mathrm{C}, 24 \mathrm{~h}$ hydrolysis method out of the majority of analytical laboratories. The next steps in the progression of protein hydrolysis will be in the automation and miniaturisation of the reaction, although there are problems of sample heterogeneity when small sample sizes of complex food products are used. Research also needs to continue into minimising hydrolysis times, increasing throughput and decreasing AA degradation. Other potential complications in the hydrolysis of proteins include the presence of numerous modified AAs, e.g. glycosylation or phosphorylation and other process-induced changes.

\section{Chromatographic separation of amino acids}

Although the chromatographic separation of AAs and their subsequent detection and quantification are two separate unit operations, in this review they have been combined as they are nevertheless still very much intertwined, and in the research discussed most practitioners use this overlap to extract synergistic advances in efficiency or sensitivity. Comprehensive reviews ${ }^{(1,2,14)}$ have covered the field up until 2008, and so this review will concentrate on technologies and methods that have been developed over the last two to 
three years. Much of the recent development has centred on Ultra HPLC combined with MS techniques, novel derivatisation reactions and new LC column developments. The relatively new field of metabolomics has resulted in the development of a number of methods for analysing AAs in various biological systems. The wider analyte range of these studies means that the AAs are just one group of compounds in any analysis. The advent of cryo-probes has also brought NMR spectroscopy within the detection limits required for the analysis of free $\mathrm{AAs}^{(2)}$ but will not be discussed in this review. Advances in AA analysers are currently running in parallel with HPLC and MS developments and so will also not be specifically reported in this review.

There has also been considerable progress in the development of direct-infusion or hyphenated MS techniques in the analysis of AAs, because MS not only matches optical detection in sensitivity, but also offers superior selectivity. Current research is largely based on further improvement, including expansion of the AA spectrum, reduction of sample preparation and analysis time, separation of underivatised AAs, automation, and synthesis of affordable isotope standards.

Although ninhydrin, phenyl isothiocyanate and o-phthaldialdehyde have been the most frequently used reagents for the pre- and post-column derivatisation of AAs, new precolumn derivatising reagents are continually being reported. The ultimate aim is for a reagent that reacts rapidly, preferably at room temperature, is stable, and has high sensitivity. The chiral nature of most of these derivatising reagents has been the basis of an excellent review ${ }^{(15)}$ which compares a number of different derivatising agents for the resolution of complex mixtures of proteinogenic D,L-AAs. An exhaustive review and derivatisation study with twenty-two AAs and simultaneous photodiode array and fluorescence detection ${ }^{(16,17)}$ has also investigated the use of 9-fluorenylmethyloxycarbonyl derivatives to analyse AAs.

Recent additions to the pre-column derivatisation stable include 2,7-dimethyl-3,8-dinitrodipyrazolo[1,5-a:1', $5^{\prime}$-d]pyrazine-4,9-dione ${ }^{(18)}$ and 2,5-dimethyl-1H-pyrrole-3,4-dicarbaldehyde ${ }^{(19)}$. These reagents react with primary amino groups, are used with a reversed phased-LC system and have limit of detection ranges of $20-80 \mathrm{pmol}$ and 3-11 pmol, respectively. The van Slyke reaction was used to form $\alpha$-hydroxy acid derivatives after reaction with dinitrogen trioxide ${ }^{(20)}$, and the derivatised AAs were then separated on an Aminex HPX-87H column eluted isocratically with $5 \mathrm{~mm} \mathrm{H}_{2} \mathrm{SO}_{4}$ and quantified by refractive index detection. A more selective and sensitive method $^{(21)}$ for trace AA determination in biological samples using the fluorescent derivatisation reagent 2-[2- $(7 \mathrm{H}$-dibenzo [a,g]carbazol-7-yl)-ethoxy] ethyl chloroformate has been used in a HPLC-fluorimetric detection-tandem MS system and had very sensitive detection limits of $0 \cdot 19-1 \cdot 17 \mathrm{fmol} / \mu \mathrm{l}$.

A combination of pre-column derivatisation with the fluorescent label 4-fluoro-7-nitro-2,1,3-benzoxadiazole and separation on a MonoClad C18-HS monolithic silica column permitted a rapid separation ( $18 \mathrm{~min})$ with high sensitivity (limit of quantification (LOQ) down to $20 \mathrm{fmol})^{(22)}$. Similarly the formation of AA butyl esters using butanolic $\mathrm{HCl}$ and their subsequent separation using ion-pair (heptafluorobutyric acid) reversed phase-LC-triple quadrupole in the multiple reaction monitoring mode resulted in enhancing sensitivity with high resolution chromatographic separation and optimal peak shapes for all AAs and a LOQ of approximately $1 \mu \mathrm{mol} / \mathrm{l}^{(23)}$.

Pre-column derivatisation with 3-aminopyridyl- $N$-hydroxysuccinimidyl carbamate and a $3 \mu \mathrm{m}$ C8 column were used to develop a high throughput 13 min cycle time HPLC-electrospray ionisation-MS method ${ }^{(24)}$. To improve throughput the derivatisation and separation steps were performed in parallel. They have also developed $p$-N,N,N-trimethylammonioanilyl $N^{\prime}$-hydroxysuccinimidylcarbamate iodide isotope-labelled internal standards to enable selected ion monitoring MS detection $^{(25,26)}$

Derivatisation has a number of inherent difficulties as mentioned above and detectors that can quantify AAs directly are becoming more prevalent. High-performance anion-exchange chromatography with integrated pulsed amperometric detection requires neither pre- or post-column derivatisation, nor oxidation of the sample and has been used in the analysis of wheat gluten AAs ${ }^{(27)}$. Pulsed electrochemical detection following HPLC exploits electrocatalytic oxidation at noble metal electrodes for the detection of polar aliphatic compounds, and provides a sensitive and direct analysis of AAs without the need for derivatisation. An indirect determination of five AAs (glutamine, glycine, methionine, phenylalanine, histidine) was developed $^{(28)}$ by using the adsorption of the AAs at the electrodes to suppress the oxidation of gluconic acid under alkaline conditions following anion exchange HPLC.

A very promising MS detection technique that is gaining increasing prominence is isotope dilution MS. The use of multiple reaction monitoring MS results in high accuracy and specificity. Presently the main limitation of the method is the availability and cost of isotope labelled AA standards. Samples may be either derivatised (e.g. with $N$-butylnicotinic acid $N$-hydroxysuccinimide ester iodide ${ }^{(29)}$ ) or underivatised (e.g. naturally abundant $13-\mathrm{C}^{(30)}$ ), and the method may also be used for the quantification of proteins based on the determination of accurate AA composition ${ }^{(31)}$. U.S. National Institute of Standards and Technology AA standards ${ }^{(32)}$ had high sensitivity, specificity, and precision with an average CV for measurements on seven different days of $3.57 \%$ (range $2 \cdot 72-4 \cdot 20 \%)$

Altering the front-end LC techniques allows for different separation selectivity. Reversed phase-LC isotope dilution MS of porcine insulin and human serum albumin hydrolysates resulted in less than 3\% expanded uncertainties and more accurate and more robust AA analysis in comparison with non-labelled methods ${ }^{(29)}$, while strong anion exchange chromatography of underivatised AAs $(0.1-0.5 \mathrm{~mm})$ had an average precision of $0.75 \%$ (range $\left.0.04-1.06 \%{ }^{(30)}\right)$. Hydrophilic interaction LC has also been coupled with isotope dilution MS to provide accurate quantification of underivatised AAs ${ }^{(33,34)}$. A zwitterion chromatography hydrophilic interaction LC column was used ${ }^{(35)}$ to analyse the AAs in hydrolysates of certified reference materials, angiotensin I and bovine serum albumin achieving LOQ values for four selected AAs between $0 \cdot 01-0 \cdot 1 \mathrm{pm}$. Better recovery and more precise data 
were obtained when compared with pre-column derivatisation with aminoquinolylhydroxysuccinimidyl carbamate. LOQ levels down to $0 \cdot 4-41 \mathrm{fmol}$ were attained for the detection of sixteen underivatised AAs in rhizosphere studies ${ }^{(36)}$ using zwitterionic-hydrophilic interaction LC separation combined with tandem MS in multiple reaction monitoring mode. Whilst the methods described above can often offer increased sensitivity this is generally not an issue in the AA analysis of foods and feeds as there is usually no concerns with sample availability.

\section{Gas chromatography-mass spectrometry}

Gas chromatography-mass spectrometry (GC-MS) has been widely used for AA analysis as it has the highest resolution of the commonly used chromatographic separation methods, and retention times, electron impact ionization and fragmentation are generally reproducible between instruments. The major limitation is the restriction to volatile analytes, and thus the need for chemical derivatisation. Silylation is the most commonly used derivativisation technique, although recent reports ${ }^{(37,38)}$ have concluded that alkylation with methyl chloroformate resulted in improved analytical performance. In a comparison of silylation using trimethylsilyl derivatives and alkylation (methyl chloroformate), the trimethylsilyl derivatisation method showed poorer reproducibility and instability during chromatographic runs. Problems of instability of derivatising reagents have largely been eliminated by in-line derivatisation.

As illustrated for the LC methods, a number of advances in the metabolomics area could be transferred to the analysis of AAs in food, including the spiking of samples with AA standards separately derivatised with deuterated derivatisation reagents, e.g. methyl chloroformate derivatisation, followed by high-throughput and sensitive GC/tandem MS to enable absolute quantification of all detected $\mathrm{AAs}^{(39)}$. In this method, positive chemical ionization was used to preserve the molecular ion and other high molecular weight fragments making selection of unique tandem MS transitions easier. High throughput automated GC/tandem MS of trimethylsilyl derivatised biological compounds using specialised libraries of mass spectra has permitted the quantification of 192 metabolites including the AAs in the urine of new-born infants ${ }^{(40)}$.

\section{Capillary electrophoresis-mass spectrometry}

Capillary electrophoresis has been used extensively for AA analysis during the past decade as capillary electrophoresis instruments have become more robust and reliable. The area has been extensively reviewed over the last six years ${ }^{(41,42)}$ and the main developments have been in improved sensitivity using derivitisation, more robust laser emitting diodes for laser-induced fluorescence and more sensitive MS detection. Recent advances in micellar electrokinetic chromatography have also been reviewed ${ }^{(43)}$, including the use of pseudostationary phases to enhance selectivity, pre-column derivatisation (e.g. 4,7-phenanthroline-5,6-dione; phanquinone) ${ }^{(44)}$, and the advantages of micellar electrokinetic chromatography over other techniques for the AA analysis. Other interesting developments include using capillary electrophoresis to determine non-protein AAs in food as indicators of food quality and safety, and the application of microchip electrophoresis to the determination of non-protein AAs in foodstuffs ${ }^{(45)}$.

\section{Other advances and applications}

Together with advances in the chemical aspects of AA analysis, advances have also been made in the associated hardware. Lab-on-chip technology in AA analysis ${ }^{(46)}$ using virtually zero-dead volume interconnections and fast mass transfer in small volume microchannels has resulted in dramatic increases in on-chip derivatisation reaction speed, while only minute amounts of sample and reagent are needed. Short channel paths allow fast subsecond separations which, together with sophisticated miniaturised detectors, can be integrated on one platform.

Recent examples of the analysis of AAs in foods include:

(1) A review of practices in the wine industry ${ }^{(47)}$ including the technique of high performance liquid magnetochromatography, where a high surface area stationary phase with paramagnetic properties $\left(\mathrm{SiO}_{2} / \mathrm{Fe}_{3} \mathrm{O}_{4}\right)$ selectively retains paramagnetic substances depending on their magnetic susceptibility in a variable intensity magnetic field $(0-5.5 \mathrm{mT})$. When AAs in samples of Spanish red and white wines ${ }^{(48)}$ were complexed with mono(1,10-phenanthroline)-Cu(II) and then analysed, this method required shorter sample processing and analysis times than other techniques, while retaining high signal reproducibility and repeatability

(2) Monitoring apple ripening ${ }^{(49)}$ using the Waters AccQ_ Fluor $^{\mathrm{TM}}$ and Waters AccQ_Tag ${ }^{\mathrm{TM}}$ pre-column derivitisation, HPLC method and

(3) The use of derivatisation-free LC with triple quadrupole tandem MS detection to demonstrate that the twentyone AAs detected in the protein-based binders of tempera paints contained an overall AA composition between that of eggs and casein ${ }^{(50)}$.

\section{Comparison of amino acid analysis methods}

The large number of reports on the development and validation of new analysis methods typically contain comparisons between the new method and a more established AA analysis method, e.g. comparison of a novel micellar electrokinetic chromatography method with a validated reference reversed phase-HPLC method ${ }^{(44)}$.

An excellent paper ${ }^{(51)}$ compared the analysis of AAs in casein and bovine serum albumin hydrolysates using an 'old' (Pico·Tag HPLC) technology to a 'new' (AccQ·Tagultra Ultra HPLC) method. The new method was considerably quicker (10 min compared with $25 \mathrm{~min}$ ) and the derivatisation was automated while maintaining a high efficiency (LOQ values were lowered from 4-9 mm to $1-6 \mathrm{~mm}$, respectively). Similarly, another study ${ }^{(52)}$ reported excellent consistency when 
analysing eighteen AAs in a human plasma pool using four LC, GC, or GC $\times$ GC separation systems with either triple quadrupole or time-of-flight MS detection systems. Isotope-labelled AA internal standards (isotope dilution) were used, samples for LCMS were underivatised and sample derivatisation for GC-MS was with N-methyl-N-[tert-butyldimethyl-silyl]trifluoroacetimide and propylchloroformate. This method was only limited by the availability of labelled AA standards.

As mentioned previously, any method must be sufficiently robust to handle the diverse range of food products presently being produced. There is a dearth of interlaboratory validation studies investigating the variability of AA methods to analyse different food groups using different AA separation and detection technologies. A two part project ${ }^{(53)}$ is currently addressing this concern; the first stage investigated the variability of AA analysis after the hydrolysis step, while the second stage will concentrate on the variability of the hydrolysis step. Five different feedstuffs or foods are being analysed by fourteen laboratories using a range of HPLC, Ultra HPLC and LC/tandem MS technologies combined with either pre- or post-derivatisation. At the midway point the preliminary results have shown that the errors observed between laboratories may be greater than the error between methods and are indeed masking the error between the methods. The researchers recommend that all laboratories should include a reference material (such as U.S. National Institute of Standards and Technology Standard Reference Materials) in every batch of samples, and that use of an internal standard should be included in the sample pretreatment phase, after hydrolysis.

The knowledge gained by these studies should be used as the first building block in the development of accurate standardised methods for analysing AAs in food proteins.

\section{Standardised methods}

To maintain consistency in reporting AA composition, e.g. for protein quality results, throughout the global food industry, and to satisfy each sovereign nation's regulatory authorities, momentum is growing to formulate and enact a defined set of standard methods for determining AA composition in foods ${ }^{(1)}$. This entails bringing together common methods for the different unit operations into one universally acceptable standard and was probably closest to realisation when ionexchange chromatography of pre- or post-column derivatised AA was the principal method used for the separation and quantification of AAs after protein hydrolysis. This need has been made even greater with the development of new methods for analysing more specific AA components and because of the increased complexity of food composition.

Although the requirement for internationally harmonised methods has been recognised by the $\mathrm{FAO}^{(57)}$, for at least the last half century there has been little action apart from an early collaborative study $^{(54)}$ on the hydrolysis of AAs from food proteins, which formed part of an AOAC International official first action for the Protein Efficiency Ratio Calculation. This was followed by two other AOAC collaborative studies ${ }^{(55,56)}$ and the establishment of the following AOAC methods:
(1) Amino Acids in Vitamin Preparations (960.47)

(2) Lysine/Available Lysine in Dietary Supplements

(3) Amino Acids/Sulphur Amino Acids in Animal Feed, Foods $(985 \cdot 28)$

(4) Amino Acids/Total Amino Acids in Fruit Juices/Lemon Juice

(5) Amino Acids in Animal Feed/Corn, Animal Feed/Poultry Meal, Animal Feed/Fishmeal

(6) Amino Acids/Lysine, Amino Acids/Methionine, Amino Acids/Threonine in Animal Feed Premixes (999·13)

Whilst the protein hydrolysis step and AA preparation in these methods is precisely defined, the AA analysis step typically has a more generic "performed by AA analyser or by cation exchange chromatography according to the suppliers instructions" description.

Indeed the $\mathrm{FAO}$ in $2003^{(57)}$ acknowledged that there was currently no official AOAC method for AA determination in foods, and that a standardised method and support for collaborative research and scientific consensus are needed to bring this about.

The European Commission ${ }^{(58)}$ has a standard (Commission Directive 98/64/EC of 3 September 1998 establishing Community methods of analysis for the determination of AAs, crude oils and fats, and olaquindox in feedingstuffs and amending Directive 71/393/EEC) "for the determination of free (synthetic and natural) and total (peptide bound and free) AAs in feedingstuffs, using an AA analyzer" that is over a decade old and, it could be argued, whilst the method is adequate for the analysis of AAs, it does not truly reflect the current scientific situation.

With the rapid advancement of analytical technologies over the last decade it is interesting to note that in its commentary on the change from total nitrogen by Kjeldahl to 'true protein' by AA analysis, in $2003 \mathrm{FAO}^{(57)}$ stated that "Its (the changes) disadvantage is that it requires more sophisticated equipment than the Kjeldahl method, and thus may be beyond the capacity of many laboratories, especially those that carry out only intermittent analyses. In addition, experience with the method is important; some AAs (e.g. the sulphur-containing AAs and tryptophan) are more difficult to determine than others". The same argument could now be applied to the adoption of the newer LCMS techniques, whereas they should be fully embraced for their sophistication and the sensitivity and unambiguity of their results.

The next phase in providing information on dietary protein quality may involve evaluation of the metabolic bioavailability of the AAs in a food ${ }^{(59)}$. A slope ratio assay utilising the indicator AA oxidation technique has recently been developed to determine the metabolic availability of AAs in pigs and humans ${ }^{(60)}$. This method addresses some of the problems associated with the slope ratio assay and could become a valuable measure of AA bioavailability in animal and human nutrition.

\section{Recommendations}

When making recommendations on a future course of action the previous FAO ${ }^{(57)}$ statement "The ultimate recommendation 
must take into account... the need to provide useful information to consumers, and the practical implications of either staying with and standardising one of the systems currently in use or moving to the other system" still very much rings true. As the research highlighted above demonstrates, there are differing degrees of robustness and sensitivity within the various protein hydrolysis and AA separation and quantification techniques available. The food industry has to ask itself what the drivers are for a standardised method, as at present it appears to be a 'horses for courses' situation.

It could be argued that the current acid hydrolysis methods are sufficient for most applications, that the weaknesses of these methods are understood and that they can be circumvented by using more exacting methods when warranted. Continuing with the equine theme 'the horse has bolted and it is too late to close the stable door' may apply with respect to the chromatography of the AAs. The advent of MS has taken the emphasis off the baseline separation of the individual AAs and, together with fast LC techniques, has allowed all AAs to be quantified with or without the need for pre- or postcolumn derivatisation.

The knowledge gained by the different inter-laboratory studies gives pointers to the most important aspects of the AA analysis methods that need to be standardised. Most of these are commonsense quality assurance factors that are routinely implemented in analytical laboratories at the present time, but which may need to be introduced into smaller laboratories. The most prudent course of action is to ensure that all necessary safeguards are in place with respect to the use of internal standards, assay proficiency programmes and quality control samples, and that each laboratory should fulfil certain 'good laboratory practice' procedures such as:

(1) Use a validated, robust method

(2) Monitor reproducibility and repeatability data

(3) Employ internal standards and quality control samples throughout the analysis

(4) Participate in an efficiency programme, e.g. Association of American Feed Control Officials programme

(5) Maintain traceable documentation for procedures, training, results and reporting.

There are still serious problems with analysing for AAs that have been modified, either post-translationally or during processing, although the newer mass spectrometric methods make the detection and quantification of these modifications possible.

Whilst there is a temptation to classify the applicability of the different technologies based on either the initial instrument costs, the required analysis throughput or even the expertise of the particular laboratory, the optimal solution would be to undertake a more extensive collaboration programme, possibly based on that undertaken by American Oil Chemists' Society/Soyabean Quality Traits, and to aim for the 'best' methods currently available, especially with respect to the separation and quantification unit operations.

This review has aimed to address the intricacies of the protein hydrolysis step, discuss what specifications or prerequisites need to be placed on the existing methods and laboratories using these methods, comment on whether one method can successfully satisfy the exacting requirements of the various unit operations, and finally pose the question 'Is there any merit in 'developing' a validated (e.g. AOAC) official method of analysis for AAs in food?'

\section{A list of recommendations has been formulated, based on these discussions:}

(1) A standardised method, based on the 'best' available at the time, should be sought and instigated.

(2) The method should be subject to regular review as new technologies and methods will supply more accurate, sensitive and 'appropriate' AA analysis.

(3) Appropriate 'checks and balances' should be included in the method; stipulate the use of internal standards, reference samples and a proficiency testing programme.

(4) The necessity for the standardised method, and its cost, must be communicated throughout the food producing nations.

\section{Recommended method:}

(1) Hydrolysis of individual AAs from the protein backbone; Protein hydrolysis: $6 \mathrm{~N} \mathrm{HCl}, 110^{\circ} \mathrm{C}, 24 \mathrm{~h}$

(2) Separation of individual AAs using a chromatographic procedure;

Chromatographic separation: Underivatised reversed phase/Hydrophilic interaction LC Ultra HPLC (RP/HILIC UHPLC)

(3) Detection and quantification of the separated AAs; Detection/quantification: Isotope dilution MS

\section{Acknowledgements}

The author states that there are no personal or financial conflicts of interest. This research received no specific grant from any funding agency in the public, commercial or notfor profit sectors.

\section{References}

1. Gilani GS, Xiao C \& Lee N (2008) Need for accurate and standardized determination of amino acids and bioactive peptides for evaluating protein quality and potential health effects of foods and dietary supplements. J AOAC Int $\mathbf{9 1}$, 894-900.

2. Kaspar H, Dettmer K, Gronwald W, et al. (2009) Advances in amino acid analysis. Anal Bioanal Chem 393, 445-452.

3. Hirs CHW, Stein WH \& Moore S (1954) The amino acid composition of ribonuclease. J Biol Chem 211, 941-950.

4. Moughan PJ \& Rutherfurd SM (2008) Available lysine in foods: a brief historical overview. J AOAC Int 91, 901-906.

5. Rutherfurd SM (2009) Accurate determination of the amino acid content of selected feedstuffs. Int J Food Sci Nutr $\mathbf{6 0}$ 53-62. 
6. Rutherfurd SM \& Gilani GS (2009) Amino acid analysis. Curr Protoc Protein Sci 58, 11.9.1-11.9.37.

7. Rutherfurd SM \& Dunn BM (2011) Quantitative amino acid analysis. Curr Protoc Protein Sci 63, 321-326.

8. Masuda A \& Dohmae N (2010) Automated protein hydrolysis delivering sample to a solid acid catalyst for amino acid analysis. Anal Chem 82, 8939-8945.

9. Buňka F, Křǐž O, Veličková A, et al. (2009) Effect of acid hydrolysis time on amino acid determination in casein and processed cheeses with different fat content. J Food Compost Anal 22, 224-232.

10. Marino R, Iammarino M, Santillo A, et al. (2010) Technical note: Rapid method for determination of amino acids in milk. J Dairy Sci 93, 2367-2370.

11. Kabaha K, Taralp A, Cakmak I, et al. (2011) Accelerated hydrolysis method to estimate the amino acid content of wheat (Triticum durum Desf.) flour using microwave irradiation. J Agric Food Chem 59, 2958-2965.

12. Messia MC, Di Falco T, Panfili G, et al. (2008) Rapid determination of collagen in meat-based foods by microwave hydrolysis of proteins and HPAEC-PAD analysis of 4-hydroxyproline. Meat Sci 80, 401-409.

13. Damm M, Holzer M, Radspieler G, et al. (2010) Microwaveassisted high-throughput acid hydrolysis in silicon carbide microtiter platforms - A rapid and low volume sample preparation technique for total amino acid analysis in proteins and peptides. J Chromatogr A 1217, 7826-7832.

14. Peace RW \& Gilani GS (2005) Chromatographic determination of amino acids in foods. J AOAC Int 88, 877-887.

15. Ilisz I, Berkecz R \& Péter A (2008) Application of chiral derivatizing agents in the high-performance liquid chromatographic separation of amino acid enantiomers: A review. J Pharm Biomed Anal 47, 1-15.

16. Jámbor A \& Molnár-Perl I (2009a) Amino acid analysis by high-performance liquid chromatography after derivatization with 9-fluorenylmethyloxycarbonyl chloride: Literature overview and further study. J Chromatogr A 1216, 3064-3077.

17. Jámbor A \& Molnár-Perl I (2009b) Quantitation of amino acids in plasma by high performance liquid chromatography: Simultaneous deproteinization and derivatization with 9-fluorenylmethyloxycarbonyl chloride. J Chromatogr A 1216, 6218-6223.

18. Gioia MG, Cacciari B, Leoni A, et al. (2006) 2,7-Dimethyl-3,8dinitrodipyrazolo[1,5-a: $1^{\prime}, 5^{\prime}$-d]pyrazine-4,9-dione: A new labelling reagent for liquid chromatographic analysis of amino acids. Anal Chim Acta 579, 152-157.

19. Gatti R, Gioia MG, Leoni A, et al. (2010) 2,5-Dimethyl-1Hpyrrole-3,4-dicarbaldehyde as a precolumn derivatization reagent for HPLC/UV detection of amino acids. $J$ Pharm Biomed Anal 53, 207-211.

20. Pleissner D, Wimmer R \& Eriksen NT (2010) Quantification of amino acids in fermentation media by isocratic HPLC analysis of their $\alpha$-hydroxy acid derivatives. Anal Chem $\mathbf{8 3}$, 175-181.

21. Li G, Cui Y, You J, et al. (2011) Determination of trace amino acids in human serum by a selective and sensitive precolumn derivatization method using HPLC-FLD-MS/MS and derivatization optimization by response surface methodology. Amino Acids 40, 1185-1193.

22. Song Y, Funatsu T \& Tsunoda M (2011) Amino acids analysis using a monolithic silica column after derivatization with 4-fluoro-7-nitro-2,1,3-benzoxadiazole (NBDF). J Chromatogr B 879, 335-340.

23. Harder U, Koletzko B \& Peissner W (2011) Quantification of 22 plasma amino acids combining derivatization and ion-pair LC-MS/MS. J Chromatogr B 879, 495-504.
24. Shimbo K, Kubo S, Harada Y, et al. (2010) Automated precolumn derivatization system for analyzing physiological amino acids by liquid chromatography/mass spectrometry. Biomed Chromatogr 24, 683-691

25. Shimbo K, Yahashi A, Hirayama K, et al. (2009a) Multifunctional and highly sensitive precolumn reagents for amino acids in liquid chromatography/tandem mass spectrometry. Anal Chem 81, 5172-5179.

26. Shimbo K, Oonuki T, Yahashi A, et al. (2009b) Precolumnderivatization reagents for high-speed analysis of amines and amino acids in biological fluid using liquid chromatography/electrospray ionization tandem mass spectrometry. Rapid Commun Mass Spectrom 23, 1483-1492.

27. Rombouts I, Lamberts L, Celus I, et al. (2009) Wheat gluten amino acid composition analysis by high-performance anion-exchange chromatography with integrated pulsed amperometric detection. J Chromatogr A 1216, 5557-5562.

28. Olson MP, Keating LR \& LaCourse WR (2009) Indirect pulsed electrochemical detection of amino acids and proteins following high performance liquid chromatography. Anal Chim Acta 652, 198-204.

29. Kinumi T, Ichikawa R, Arimoto H, et al. (2010) Traceable amino acid analyses of proteins and peptides by isotopedilution mass spectrometry using precolumn derivatization reagent. Anal Sci 26, 1007-1010.

30. Abaye DA, Morrison DJ \& Preston T (2011) Strong anion exchange liquid chromatographic separation of protein amino acids for natural 13C-abundance determination by isotope ratio mass spectrometry. Rapid Commun Mass Spectrom 25, 429-435.

31. Munoz A, Kral R \& Schimmel H (2011) Quantification of protein calibrants by amino acid analysis using isotope dilution mass spectrometry. Anal Biochem 408, 124-131.

32. Woolfitt AR, Solano MI, Williams TL, et al. (2009) Amino acid analysis of peptides using isobaric-tagged isotope dilution LC - MS/MS. Anal Chem 81, 3979-3985.

33. Lafaye A, Junot C, Pereira Y, et al. (2005a) Combined proteome and metabolite-profiling analyses reveal surprising insights into yeast sulphur metabolism. J BiolChem 280, 24723-24730.

34. Lafaye A, Labarre J, Tabet J-C, et al. (2005b) Liquid chromatography-mass spectrometry and $15 \mathrm{~N}$ metabolic labeling for quantitative metabolic profiling. Anal Chem 77, 2026-2033.

35. Kato M, Kato H, Eyama S, et al. (2009) Application of amino acid analysis using hydrophilic interaction liquid chromatography coupled with isotope dilution mass spectrometry for peptide and protein quantification. $J$ Chromatogr $B$ 877, 3059-3064.

36. Dell'mour M, Jaitz L, Oburger E, et al. (2010) Hydrophilic interaction LC combined with electrospray MS for highly sensitive analysis of underivatized amino acids in rhizosphere research. J Sep Sci 33, 911-922.

37. Smart KF, Aggio RBM, Van Houtte JR, et al. (2010) Analytical platform for metabolome analysis of microbial cells using methyl chloroformate derivatization followed by gas chromatography-mass spectrometry. Nat Protoc 5, 1709-1729.

38. Villas-Bôas SG, Smart KF, Sivakumaran S, et al. (2011) Alkylation or silylation for analysis of amino and non-amino organic acids by GC-MS? Metabolites 1, 3-20.

39. Kvitvang HFN, Andreassen T, Adam T, et al. (2011) Highly sensitive GC/MS/MS method for quantitation of amino and nonamino organic acids. Anal Chem 83, 2705-2711.

40. Shoemaker JD (2010) One-step metabolomics: Carbohydrates, organic and amino acids quantified in a single procedure. J Vis Exp 40, available at: http://www.jove. $\mathrm{com} /$ index/Details.stp?ID $=2014$. 
41. Poinsot V, Rodat A, Gavard P, et al. (2008) Recent advances in amino acid analysis by CE. Electrophoresis 29, 207-223.

42. Poinsot V, Gavard P, Feurer B, et al. (2010) Recent advances in amino acid analysis by CE. Electrophoresis 31, 105-121.

43. Iadarola P, Ferrari F, Fumagalli M, et al. (2008) Determination of amino acids by micellar EKC: recent advances in method development and novel applications to different matrices. Electrophoresis 29, 224-236.

44. Gotti R, Gioia MG, Gatti R, et al. (2006) Phanquinone as a suitable derivatization reagent in micellar electrokinetic chromatography and HPLC analysis of amino acids. $J$ Sep Sci 29, 1259-1267.

45. Castro-Puyana M, Crego AL, Marina ML, et al. (2007) CE methods for the determination of non-protein amino acids in foods. Electrophoresis 28, 4031-4045.

46. Pumera M (2007) Microfluidics in amino acid analysis. Electrophoresis 28, 2113-2124.

47. Callejón RM, Troncoso AM \& Morales ML (2010) Determination of amino acids in grape-derived products: A review. Talanta 81, 1143-1152.

48. Barrado E, Rodriguez JA \& Castrillejo Y (2009) Determination of primary amino acids in wines by high performance liquid magneto-chromatography. Talanta 78, 672-675.

49. Zhang Y, Li P \& Cheng L (2010) Developmental changes of carbohydrates, organic acids, amino acids, and phenolic compounds in 'Honeycrisp' apple flesh. Food Chem 123, $1013-1018$

50. Zangrando R, Piazza R, Cairns WR, et al. (2010) Quantitative determination of un-derivatised amino acids in artistic mural paintings using high-performance liquid chromatography/ electrospray ionization triple quadrupole mass spectrometry. Anal Chim Acta 675, 1-7.

51. Boogers I, Plugge W, Stokkermans YQ, et al. (2008) Ultraperformance liquid chromatographic analysis of amino acids in protein hydrolysates using an automated precolumn derivatisation method. J Chromatogr A 1189, 406-409.
52. McGaw EA, Phinney KW \& Lowenthal MS (2010) Comparison of orthogonal liquid and gas chromatography-mass spectrometry platforms for the determination of amino acid concentrations in human plasma. J Chromatogr A 1217, 5822-5831.

53. Simpson JV, Zhang Y, Cantrill R, et al. (2009) AOCS/SQT Amino Acid Round Robin Study. www.aafco.org/Portals/0/ labmethods/5-lab_aa_round_robin.pdf.

54. Satterlee LD, Kendrick JG, Marshall HF, et al. (1982) In vitro assay for predicting protein efficiency ratio as measured by rat bioassay: collaborative study. J Assoc Off Ana. Chem 65, 798-815.

55. MacDonald JL, Krueger MW \& Keller JH (1985) Oxidation and hydrolysis determination of sulfur amino acids in food and feed ingredients: collaborative study. $J$ Assoc Offic Anal Chem 68, 826-829.

56. Allred MC \& MacDonald JL (1988) Determination of sulfur amino acids and tryptophan in foods and food and feed ingredients: collaborative study. I Assoc Offic Anal Chem 71, 603-606.

57. Food and Agriculture Organisation (2003) Food energy methods of analysis and conversion factors: report of a technical workshop. In FAO Food and Nutrition Paper 77. Rome: WHO.

58. European Commission (1998) Commission Directive 98/64/ EC of 3 September 1998: Establishing Community methods of analysis for the determination of amino-acids, crude oils and fats, and olaquindox in feedingstuffs, and amending Directive 71/393/EEC. Offic J Eur Communities L257, 14-23.

59. Food and Agriculture Organisation (2007) Protein and amino acid requirements in human nutrition: report of a joint $\mathrm{FAO} /$ WHO/United Nations University expert consultation. In WHO Technical Report Series no. 935. Geneva: WHO.

60. Levesque CL, Moehn S, Pencharz PB, et al. (2010) Review of advances in metabolic bioavailability of amino acids. Livestock Sci 133, 4-9. 